\title{
3D Reconstruction of Scattered Point-cloud Using RBF
}

\author{
Tian Chengjun $^{{ }^{*}}$, Yang Qinglong ${ }^{b}$, Zhang Chenjie ${ }^{c}$ \\ School of Electronics and Information Engineering \\ Changchun University of Science and Technology, Changchun 130022, China

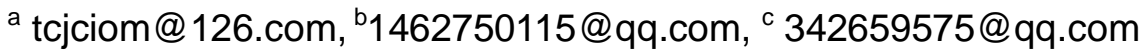

Keywords: point-cloud, Principal component analysis algorithm, radial basis function

Abstract. Reverse engineering is an important way to transmit information in modern manufacture technology. Reverse engineering technology widely applied in the automobile and aviation industry. Surface reconstruction of scattered point-cloud is a key part in the operation process of reverse engineering. Firstly, the dissertation analyzed the local attributes of the scattered point-cloud and researched the normal vector evaluation and direction consistency with the principal component analysis algorithm. Secondly, the implicit surface reconstruction algorithm is analyzed and compared, the radial basis function is chosen as the reconstruction method, and the $3 \mathrm{D}$ reconstruction of scattered point-cloud is realized. The algorithm not only reduces the time and space requirements of surface fitting, but also repairs the hole in the point-cloud.

\section{Introduction}

With the rapid development of computer technology, especially the rapid development of digital measurement technology, reverse engineering technology has been widely used in aviation, medical, cultural heritage protection and other fields. One of the most important problems in reverse engineering is the reconstruction of three-dimensional surface, which is also the core and hot spot in the research of reverse engineering. Because of the limited sample density, partial information of the real object has lost in the discrete point-cloud obtained by laser scanning, so the object's geometry can not be completely described. In order to obtain the geometric shape of the measuring object, we must fit the continuous surface of the local geometrical features of the physical entity by means of a discrete sampling data point set. The purpose of this dissertation is to construct the implicit surface from the scattered point-cloud and transform it into a three-dimensional grid.

\section{Point-cloud collection}

Point-cloud data acquisition of the entity is the first step of reverse engineering and it is the basis of reverse engineering technology to collect the surface point-cloud of the entity with high efficiency and precision. The step is mainly used to establish the digital information of the geometrical surface of the object by the way of 3D scanning mode, each point contains 3D coordinates, some may contain color information or intensity information [1]. Using these discrete point-cloud data can obtain the surface shape of the actual object by interpolation, dense point-cloud can build a more accurate model. The measurement method of the point-cloud in reverse engineering can be divided into two kinds: contact type, non-contact type[1]. Contact type three dimensional measurement is mainly through the actual contact with the surface of the object to calculate the depth, which depends on the coordinate measuring machine now. The basic principle of non-contact measurement method is to calculate the geometrical information of the object by measuring the energy emitted by the device, combine the energy of the reflected back from the measured work-pieces, using the method of flight time and image processing to calculate the $2 \mathrm{D}$ 
tomographic image or point-cloud data. As is shown in Fig. 1, the point-cloud data obtained by laser radar.

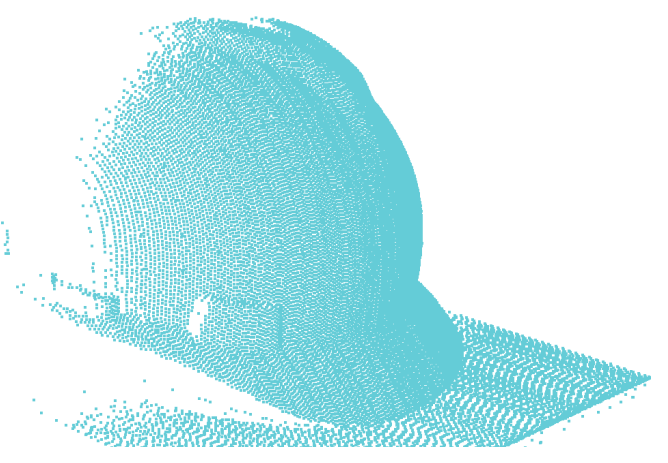

Fig. 1 Scattered point-cloud

\section{Normal vector estimation of scattered point-cloud}

The normal vector is a very important attribute in the representation of points, and a lot of point based rendering methods are dependent on the accurate normal of sampling points. First of all, to establish sampling grids of data points, and then calculate the weighted average of all adjacency triangles normal to obtain a vertex normal. This method is a global computing method. At present, the number of 3D point-cloud obtained by laser scanning is likely to be one hundred thousand or even a million. It is difficult to build a global grid in such a large amount of data. Therefore, this dissertation uses the method of analyzing the local attributes of the point-cloud to obtain the normal vector information of the sample data points[2,3]. The estimation of normal vector has the following methods:

(1) The least square method is used to fit a plane of the sampling points $p_{i}$ and its $\mathrm{K}$ neighborhood

points $\left(p_{i 1,} p_{i 2,} p_{i 3,} \mathrm{~L} p_{i k}\right)$, then the sampling node $p_{i}$ and $n_{i}$ can be used to replace the normal vector of the fitting plane.

(2)The moving least square method is used to estimate the hyper plane of the point, its normal is used as the normal of the sampling point. The method is very accurate and needs to solve the nonlinear equation.

(3)Principle component analysis of the $p_{i}$ and its $\mathrm{K}$ fields is carried out. First of all, the covariance matrix of the sampling point and the nearest neighbor point is defined. Then the eigenvalues and eigenvectors of the matrix are solved, and the normal vector of the sample point is the feature vector corresponding to the minimum eigenvalues. The method is sample, easy to operate, and the effect is good. It can be used to estimate the normal vector of scattered point cloud. $P=\left\{p_{i} \in R^{3}, i=1,2, \ldots, N\right\}$ is a scattered data point obtained from the surface of the measured entity. Then each data point $\left\{p_{i}=x_{i}, y_{i}, z_{i}\right\}$ corresponds to a tangent plane $T_{p}\left(P_{i}\right)$, it can be used as the local linear approximation at here. The tangent plane corresponding to each data point can be represented by the center of mass of the tangent plane $o_{i}=\sum_{j=1}^{k} \frac{p_{i k}}{k}$ and the unit normal vector $n_{i}$, where $p_{i k}$ is the $\mathrm{K}$ nearest neighbor point of $p_{i}$. The normal vector of point $p_{i}$ can be calculated by principal component analysis. Firstly, using the $\mathrm{K}$ nearest neighbor points to establish the covariance matrix, as is shown in formula1, the matrix is a $3 * 3$ semi positive definite symmetric 
matrix, which describes the distribution of the statistical properties of the sample point $p_{i}$.

$$
\operatorname{COV}=\left[\begin{array}{ccc}
\sum\left(x_{j}-o_{x}\right)^{2} & \sum\left(x_{j}-o_{x}\right)\left(y_{j}-o_{y}\right) & \sum\left(x_{j}-o_{x}\right)\left(z_{j}-o_{z}\right) \\
\sum\left(y_{j}-o_{y}\right)\left(x_{j}-o_{x}\right) & \sum\left(y_{j}-o_{y}\right)^{2} & \sum\left(y_{j}-o_{y}\right)\left(z_{j}-o_{z}\right) \\
\sum\left(z_{j}-o_{z}\right)\left(x_{j}-o_{x}\right) & \sum\left(y_{j}-o_{y}\right)\left(z_{j}-o_{z}\right) & \sum\left(z_{j}-o_{z}\right)^{2}
\end{array}\right]
$$

After working out the corresponding units feature vector $v_{1}, v_{2}, v_{3}$ of the semi positive definite matrix and three eigenvalues $\lambda_{1}, \lambda_{2}, \lambda_{3}$, the direction of the micro tangent plane is parallel to the minimum eigenvalues of the normal vector. Therefore, the normal vector of the data points can be determined by calculating the eigenvalues of the covariance matrix, if $\lambda_{1}<\lambda_{2}<\lambda_{3}$, then $n_{i}=v_{1}$ or $n_{i}=-v_{1}[4,5]$.

From the above discussion we know that there is a unit normal vector of a micro tangent plane in the point-cloud data. But the normal vector estimated by PCA method, maybe points to different direction: it is in agreement with the normal direction of the surface or the opposite direction. Therefore, it is necessary to adjust the normal vector direction of the micro cut plane set so that all the normal vectors are unified to the inner or outer of the reconstructed surface, as is shown in Fig. 2, the normal vector of the direction of the non-adjustment is estimated. If the reconstructed surface is smooth and the density of point-cloud data is large about the adjacent scattered point-cloud, then the tangent plane of these scattered data points is almost parallel and the dot product of their corresponding normal vector is $n_{i} \cdot n_{j}= \pm 1$. If two adjacent tangent planes normal vectors are in the same direction, then $n_{i} \cdot n_{j}=1$. If two adjacent tangent planes normal vectors are in the opposite direction, then $n_{i} \cdot n_{j}=-1$. So the estimation of the normal vector is to be reversed, the method can be used to adjust the direction of point-cloud vector. As is shown in Fig. 3, the method is used to adjust the direction of the normal vector[10].

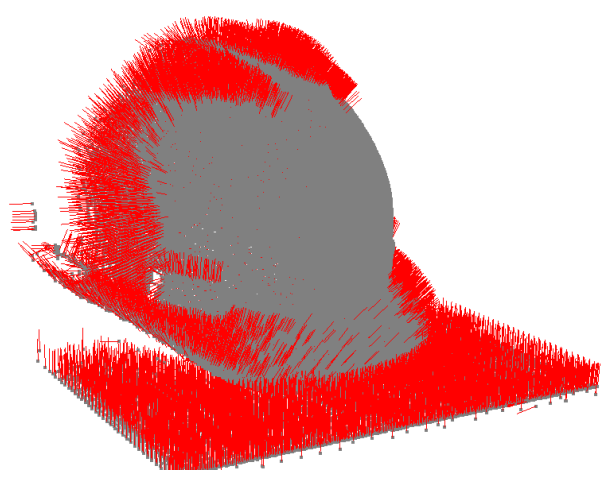

Fig.2 non-adjustment direction normal vector

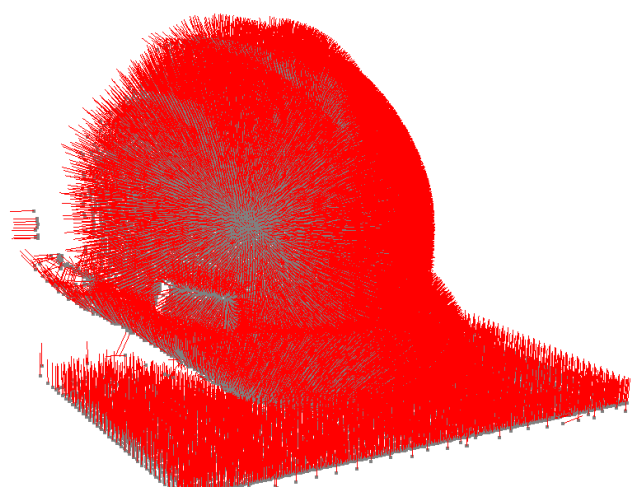

Fig.3 direction adjustment normal vector

\section{$3 D$ reconstruction of scattered point-cloud using $R B F$}

In the field of reverse engineering, modeling of the free form surface is one of the core issues of the researchers. The surface based on sampling point is a dense collection of discrete sampling points, which is represented by a multi-resolution organization on continuous curved surface. It is the boundary representation of the information represented by the model[6]. The sampling points in the 3D space $\left\{p_{1}, p_{2, \mathrm{~L}}, p_{n} \in R^{3}\right\}$ are distributed in the outer surface of the measured entity, firstly, the discrete sampling points are assumed to satisfy the necessary sampling conditions, and these sampling points are completely covered by topological and geometrical information of the measured entity. Therefore, the representation of the point based model is a surface representation method, the purpose is to construct a continuous surface from the discrete point. Hoppe put the problem in general and propose a unified definition: The so-called surface reconstruction is to use a 
sample set $p$ and sample processing of some information(such as the size of error and the sampling density) to determine a surface $S$ ', which can be reasonably approximated by an unknown surface $S[8]$.

In the method of implicit surface reconstruction, the radial basis function method is one of the most widely used methods. At present, the research on the radial basis function has received more and more attention, and foreign scholars have made great achievement using RBF interpolation to solve 3D surface reconstruction and display. The basic principle of radial basis function interpolation is analyzed in this section and the radial basis function is used to reconstruct the 3D scattered point-cloud.

The interpolation problem is described as follows, a collection of different data points with $\mathrm{N}$ is given $\left\{X_{j} \in \mathfrak{R}^{m}, j=1,2,3, \mathrm{~K}, N\right\}$, as well as a collection of $\mathrm{N}$ real numbers $\left\{f_{j} \in \mathfrak{R}, j=1,2,3, \mathrm{~K}, N\right\}$, to find a function: $S: \mathfrak{R}^{m} \rightarrow \mathfrak{R}$, satisfy the interpolation conditions as follows:

$$
f\left(X_{j}\right)=f_{j}, j=1,2,3, \mathrm{~K}, N
$$

Obviously, if $\mathrm{m}=3$, all the given data points are defined by $S$.Radial basis function method is one of the effective way to solves the above interpolation problem. Using RBF interpolation method can be summarized as the following form of the basis functions: $\phi: \mathfrak{R}^{m} \rightarrow \mathfrak{R}$, then function satisfied:

$$
f(X)=\sum_{j=1}^{N} \omega_{j} \phi\left(\left\|X-X_{j}\right\|\right)
$$

In formula $3, c_{j}$ is undetermined coefficient, $\phi$ is radial basis function, $\|\bullet\|$ is the Euclidean norm, given the $\mathrm{N}$ data points $\left\{X_{j} \in \mathfrak{R}^{m}, j=1,2,3, \mathrm{~K}, N\right\}$ are the centers of the radial basis functions. As is shown in Fig. 4, the model can be obtained by using the radial basis function fitting. The method not only realized 3D reconstruction, but also filled the small holes.

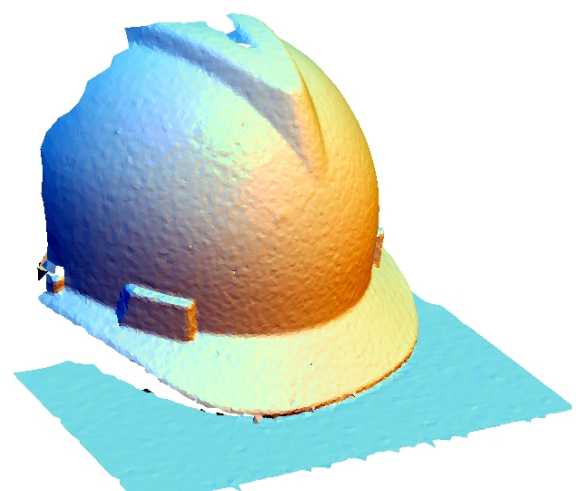

Fig. 4 RBF fitting 3D surface

\section{Conclusion}

The dissertation analyzed the basic principle of the scattered point-cloud collection, for the requirement of the normal vector of the scattered data points in the $3 \mathrm{D}$ reconstruction using implicit function, the local attributes of the scattered point-clouds are analyzed with principal component analysis algorithm. By solving the covariance matrix of the scattered point-cloud, the normal vector estimation value of point-cloud method is obtained and the direction adjustment error of the normal vector is eliminated. The radial basis function is used to reconstruct the surface of scattered point-cloud, the method can realize the reconstruction of non-homogeneous distribution scattered 
point-cloud data, and it has a good adaptability.

\section{Acknowledgements}

The research project is supported by the Education Department of Jilin Province scientific research project (No. 22210329)

\section{Reference}

[1] Vinesh Raja. Reverse Engineering: An Industrial Perspective, Springer-Verlag London Limited, 2008, p.1-5.

[2]KE Ying Lin. Study of CAD Modeling for Reverse Engineering, Journal of Computer-Aided Design \& Computer, 2001,13: p.1-6.

[3]LI Bao, Survey on normal estimation for 3D point clouds. Computer Engineering and Applications, 2010,46 (23): p.1-7.

[4]Zhang fan, On Geometry Processing of Point Cloud Data: [Doctoral Dissertation], Xian: Northwest University, 2013.

[5]OuYang D, Feng HY. On the normal vector estimation for point cloud data from smooth surfaces [J], Computer-Aided Design, 2005, 37(10):p.1071-1079.

[6]Pauly M, Gross M, Efficient Simplification of Point-Sampled Surfaces [A], IEEE Visualization Boston, MA, USA, 2002, 27, p.163-170.

[7] Lu Zhangping, Point Cloud Registration Algorithm Based on Free-form Surface[J], Journal of Jiangsu University, 2015.4.

[8] H. Hoppe, T. DeRose, T. Duchamp. Surface reconstruction from unorganized points, Computer Graphics (SIGGRAPH'92 Proceedings).1992, 26 (2):p71-78.

[9]O. Shall, Surface from Scattered Points: A Brief Survey of Recent Developments, In First International Workshop on Semantic Virtual Environments ,Switzerland.(2005), p.138-147

[10]Renka RJ, Multivariate Interpolation of Large Sets of Scattered Data[J], ACM Transactions on Mathematical Software, June, 1988, 14(2):p.139-148 